\title{
An exclusion map for Von Recklinghausen neurofibromatosis
}

\author{
MANSOOR SARFARAZI*, SUSAN M HUSON†, AND \\ JOHN H EDWARDS $\ddagger$ \\ From *the Institute of Medical Genetics, University of Wales College of Medicine, Heath Park, Cardiff \\ CF4 4XN; t the Division of Inherited Metabolic Diseases, Clinical Research Centre, Northwick Park Hospital, \\ Harrow, Middlesex HAl 3UJ; and $\ddagger$ the Genetics Laboratory, Department of Biochemistry, University of \\ Oxford, South Parks Road, Oxford OX1 3QU.
}

SUMmaRY By using all the genetic linkage data available between Von Recklinghausen neurofibromatosis (VRNF) and various loci on the autosomes, a graphical display of all the non-excluded areas is presented. The probability of VRNF being on any of the 22 autosomes is also shown. The exclusion map presented is for 90 markers that have been localised relatively accurately. Data are also presented for a further 24 markers that have not yet been adequately localised. This exclusion map shows that most of the genome has been excluded as a likely location of any locus responsible for this disorder in the majority of families. Chromosomes 5, 10, 17 , and 18 remain largely unexcluded. Concentration on these parts of the genome should help in the identification of the site of the VRNF gene.

The mapping of the gene for Von Recklinghausen neurofibromatosis (VRNF) is of paramount importance to our eventual understanding of the disease pathogenesis. It is one of the commonest autosomal dominant disorders in man and the very varied disease complications cause significant morbidity and mortality. ${ }^{1}$ In 1986 , we presented a preliminary exclusion map for VRNF using the computer programme EXCLUDE, based on the results of the Cardiff group and published linkage reports up to that time. ${ }^{2}$

More recently (February 1987), all groups involved in linkage analysis of VRNF were invited to attend the first European Symposium on Neurofibromatosis sponsored by the British neurofibromatosis patients' association, LINK. At the meeting it was agreed that a significant advance towards the mapping of the gene would be made by the pooling of all linkage data for VRNF to date. In particular, this would avoid unnecessary reduplication of negative results and highlight those areas of the genome not yet studied.

As a result of this meeting a consortium of those involved with VRNF linkage was formed. We are privileged to present in this paper a more detailed

Received for publication 30 April 1987 Accepted for publication 5 May 1987. exclusion map for VRNF based on previously published reports, and on the data provided by other members of the consortium; the individual data sets are given in full in the subsequent papers in this issue of the Journal.

\section{Description of programme 'EXCLUDE'}

The programme EXCLUDE is written in Pascal (Turbo version) and is designed to calculate the likelihood distribution of an unplaced locus around a series of previously mapped loci, ${ }^{3}$ following the general principle of Cook et al, ${ }^{4}$ who first used the term 'exclusion mapping'. The likelihood distribution at various recombination fractions or map distances gives the relative chance that the locus is in that position. In the pairwise analysis when the counts of recombinants and non-recombinants are available, the peak and position of the likelihood distribution are uniquely defined by these counts. However, when the direct counts are not available, an approximation may be obtained by defining the 'equivalent observations' 5 after the data have been analysed by the likelihood method of Morton. ${ }^{6}$ This is achieved by defining the number of recombinants and non-recombinants which would give the same height and position of the likelihood distribution. For a given value of recombination fraction $(\theta)$ and 
the corresponding value of lod score $(Z)$, the equivalent number of informative meioses (s) is defined as:

$$
\mathrm{s}=\mathrm{Z} /\left[\theta^{*} \log (2 \theta)+(1-\theta)^{*} \log (2(1-\theta))\right]
$$

and the equivalent number of recombinants will be:

$$
r=\theta^{*} s
$$

By knowing $r$ and $s$, the likelihood for each position is then computed directly from these equivalents. For codominants and close loci, almost all the data can be extracted by counting.

The distribution of recombinant events varies by sex, age, chromosome number, and by position on that chromosome. In linkage data relating to a disease, only part of the information is usually available in the form of recombinants and nonrecombinants, and the rest of the data has to be extracted in the form of lod score distributions for various values of $\theta$. The direct count of recombinants and non-recombinants provides the optimal way of estimating the maximum likelihood. This also has the advantages of simplicity, additivity, and directness of meaning.

As the relationship between the recombination fraction $(\theta)$ and the map distance $(w)$ is not linear, this leads to some problems in the positional likelihood distribution. By definition, $\theta$ and $\mathrm{w}$ are equal when $\theta=0.01$, and approximately linear up to $\theta=0.05$; however, $w$ progressively exceeds $\theta$ at higher values. It is simplest to use Haldane's function, although it assumes two strand meiosis and an equal chance of recombination at any point unrelated to any nearby recombination. It is also possible to use Kosambi's mapping function in the EXCLUDE programme. ${ }^{3}$

The EXCLUDE programme finds the peak likelihood distribution by parabolic interpolation when presented with $\mathrm{Z}$ scores. When the information cannot be extracted efficiently by counting, the approximation is usually close, as is easily demonstrated by defining these 'equivalent observations' and then computing the lods from $\theta$. All likelihood data can be converted into these equivalents. The overall positional likelihoods are combined by multiplication and standardised either to display a unit area or to 'fill' the diagram.

The programme accepts pairwise data and is inefficient for close linkage, but the information loss is small when no linkage is established.

\section{Origin of the linkage data}

Up to now, more than 10 groups have undertaken genetic linkage studies to map the locus of the
VRNF gene, but none of these has detected significant linkage between a marker and the dis=order. A total of 114 markers on the variou药 autosomes has been tested for a possible linkage to VRNF. Out of these, 90 have been localise firmly with various degrees of precision, 18 ar known to be on a specific chromosome, and the्ष remaining six have not been assigned to ang chromosome.

All the linkage data from previously published work and from those participating in this consortium. were pooled to be used with the programme् EXCLUDE. When the lod score was uniformls negative for all values of $\theta$, the lod at $0 \cdot 10$ was used For $Z>0$ the value of $Z_{\max }$ and the value of $\theta$ aंt which it occurred were used. The overall linkage्己 data available on VRNF are summarised in tables fr and 2. The chromosomal position in table $1 \overrightarrow{\vec{G}}$ denoted by chromosome number, a period, and the location of the marker on that chromosome expressed as \% of pter to qter. For example, the value of 1.36 for PGM1 means that this marker is of chromosome 1 at an approximate position of $36^{\circ} \%$ from the top of that chromosome. When the localisation of a marker on the chromosome is expanded over a region of that chromosome, the location of that marker is taken to be in the migelie of that region. For those markers with no region localisation, the data were excluded and are sherien in the first part of table 2. All the unassigne markers were also excluded from the data set an are shown in the lower part of table 2 .

Input to programme EXCLUDE consists of chromosomal position, locus name, a number defin $\overrightarrow{\overrightarrow{0}}$ ing the types of data ( 1 for this data set), followed b the linkage data given in the form of $\theta$ and $\mathrm{Z}$ (fir five columns of table 1). Each of these fields separated by a few spaces. As the programme starks to read each of the data sets on a separate line, calculates the equivalent number of informative meioses (s), number of recombinants ( $r$ ), and the ratio $\mathrm{r} / \mathrm{s}$ as described previously (table 1 ). The programme then accesses an internal file (HUMAN.CHR) which contains the approximat? length of each of the human chromosomes given im centimorgans for both male and female chromo. somes, which is assumed proportional to lengtwo as a rough approximation. The programme then calculates the positional likelihood of the disease locus on each chromosome, and also the peto centage probability of any locus being on an耳 of the 22 autosomes (table 3). The last twe columns of table 3 give the maximum values of likelihood and probability of VRNF being on the? chromosome. The figure is the graphical reprev sentation of these values. 
TABLE 1 Overall linkage data used to obtain the VRNF exclusion map. The first five columns of data were used as input to the programme.

\begin{tabular}{|c|c|c|c|c|c|c|c|c|}
\hline $\begin{array}{l}\text { Position } \\
\text { on the } \\
\text { chromosome }\end{array}$ & Locus & $\begin{array}{l}\text { Data } \\
\text { type }\end{array}$ & $\begin{array}{l}\text { Theta } \\
\text { (cM) }\end{array}$ & $\begin{array}{l}\text { Lod } \\
\text { score }\end{array}$ & Localisation & $r$ & $s$ & Reference \\
\hline $1 \cdot 05$ & $P G D$ & 1 & $0 \cdot 10$ & -0.44 & $1 \mathrm{p} 36 \cdot 2 \rightarrow \mathrm{p} 36 \cdot 13$ & 0.99 & 1.98 & 9 \\
\hline 1.09 & $R H$ & 1 & $0 \cdot 10$ & $-10 \cdot 71$ & $1 \mathrm{p} 36 \cdot 2 \rightarrow \mathrm{p} .34$ & $24 \cdot 14$ & $48 \cdot 28$ & 9.11 .16 \\
\hline $1 \cdot 24$ & $D I$ & 1 & $(0 \cdot 10$ & $-3 \cdot(1)$ & $1 \mathrm{p}$ & $6 \cdot 80$ & $13 \cdot 61$ & 20 \\
\hline $1 \cdot 36$ & PGMI & 1 & $0 \cdot 10$ & -3.74 & $1 \mathrm{p} 22 \cdot 1$ & $8 \cdot 43$ & $16 \cdot 86$ & 9.11 .16 \\
\hline $1 \cdot 36$ & $N G F B$ & $i$ & $(0 \cdot 10$ & -5.98 & $1 \mathrm{p} 22 \cdot 1$ & $13 \cdot 48$ & $26 \cdot 96$ & 12. 19 \\
\hline $1 \cdot 39$ & $A M Y I$ & 1 & $0 \cdot 10$ & 0.74 & $1 \mathrm{p} 21$ & 0.46 & $4 \cdot 63$ & 10 \\
\hline $1 \cdot 53$ & $F Y$ & 1 & $0 \cdot 10$ & $-11 \cdot 31$ & $1 \mathrm{p} 21 \rightarrow \mathrm{q} 23$ & $25 \cdot 49$ & 50.98 & 9.11 .16 \\
\hline $1 \cdot 72$ & $F 13 B$ & 1 & $0 \cdot 10$ & $-1 \cdot 83$ & $1 \mathrm{q}$ & $4 \cdot 12$ & 8.25 & 18 \\
\hline 1.72 & DI & 1 & $0 \cdot 10$ & $-2 \cdot 20$ & 19 & 4.96 & 9.92 & 20 \\
\hline $1 \cdot 75$ & AT3 & 1 & $0 \cdot 20$ & 0.57 & $1 \mathrm{q} 23 \longrightarrow \mathrm{q} 25$ & $1 \cdot 36$ & $6 \cdot 81$ & 17. 18 \\
\hline $2 \cdot 06$ & $A C P I$ & 1 & $0 \cdot 10$ & -4.59 & $2 \mathrm{p} 25$ or $2 \mathrm{p} 23$ & $10 \cdot 35$ & $20 \cdot 69$ & $10.11,16$ \\
\hline $2 \cdot 08$ & POMC & 1 & $0 \cdot 10$ & $-2 \cdot 09$ & $2 \mathrm{p} 23$ & $4 \cdot 71$ & $9 \cdot 42$ & 22 \\
\hline $2 \cdot 29$ & $T G F A$ & 1 & $0 \cdot 10$ & -1.47 & $2 \mathrm{p} 13$ & $3 \cdot 31$ & 6.63 & 18 \\
\hline $3 \cdot 07$ & $R A F I$ & 1 & $0 \cdot 10$ & $-3 \cdot 18$ & $3 p 25 \rightarrow p 24$ & $7 \cdot 17$ & $14 \cdot 33$ & 19 \\
\hline $3 \cdot 23$ & $D I S I$ & 1 & $0 \cdot 10$ & $-4 \cdot(17$ & $3 \mathrm{p} 21$ & $9 \cdot 17$ & $18 \cdot 35$ & 18 \\
\hline $3 \cdot 25$ & $D 3 S 2$ & 1 & $(0 \cdot 10$ & $-2 \cdot 12$ & $3 \mathrm{p} 14 \rightarrow \mathrm{p} 21$ & $4 \cdot 78$ & $9 \cdot 56$ & 22 \\
\hline $3 \cdot 26$ & D3S3 & 1 & $0 \cdot 10$ & $-2 \cdot 01$ & $3 q$ & 4.53 & $9 \cdot 06$ & 22 \\
\hline $3 \cdot 74$ & $T F$ & 1 & $0 \cdot 10$ & -0.21 & $3 q 21 \rightarrow q 26 \cdot 1$ & 0.47 & 0.95 & 16 \\
\hline $4 \cdot 08$ & $R A F 2$ & 1 & $0 \cdot 10$ & $-2 \cdot 49$ & 4 p15 $\rightarrow$ pter & $5 \cdot 61$ & $11 \cdot 22$ & 19 \\
\hline $4 \cdot 20$ & $D 4 S I$ & 1 & $0 \cdot 10$ & -0.27 & $4 \mathrm{p} 15 \cdot 1 \rightarrow \mathrm{q} 11$ & 0.61 & $1 \cdot 22$ & 19 \\
\hline $4 \cdot 22$ & D4S35 & 1 & $0 \cdot 10$ & $-11 \cdot 27$ & $4 \mathrm{p} 15 \cdot 1 \rightarrow \mathrm{q} 12$ & $25 \cdot 40$ & $50 \cdot 80$ & 18.19 \\
\hline $4 \cdot 32$ & $G C$ & 1 & $0 \cdot 10$ & $-14 \cdot 43$ & $4 \mathrm{q} 12 \rightarrow \mathrm{q} 13$ & $32 \cdot 52$ & $65 \cdot(14$ & $7.9,11,14,16,18$ \\
\hline $4 \cdot 33$ & $A L B$ & 1 & $0 \cdot 10$ & -1.42 & $4 q 11 \rightarrow q 13$ & $3 \cdot 20$ & $6 \cdot 40$ & 18.19 \\
\hline $4 \cdot 35$ & $M T 2 P I$ & 1 & $0 \cdot 10$ & $-1 \cdot 37$ & $4 \mathrm{p} 11 \rightarrow \mathrm{q} 21$ & $3 \cdot 09$ & $6 \cdot 18$ & 22 \\
\hline $4 \cdot 48$ & $A D H 3$ & 1 & $0 \cdot 30$ & 0.21 & $4 \mathrm{q} 21 \rightarrow \mathrm{q} 25$ & $1 \cdot 76$ & $5 \cdot 88$ & 18 \\
\hline $4 \cdot 61$ & $E G F$ & 1 & $0 \cdot 10$ & $-1 \cdot 36$ & $4 \mathrm{q} 25 \rightarrow \mathrm{q} 27$ & $3 \cdot 07$ & $6 \cdot 13$ & 18 \\
\hline $4 \cdot 66$ & $F G$ & 1 & $0 \cdot 10$ & -0.25 & $4 q_{26} \rightarrow q_{2} 8$ & 0.56 & $1 \cdot 13$ & 18 \\
\hline $4 \cdot 73$ & GIFN & 1 & $0 \cdot 10$ & $-1 \cdot 15$ & $4 q$ & $2 \cdot 59$ & $5 \cdot 18$ & 18 \\
\hline $4 \cdot 75$ & MNSS & 1 & $0 \cdot 10$ & $-10 \cdot 63$ & $4 \mathrm{q} 28 \rightarrow \mathrm{q} 31$ & $23 \cdot 96$ & $47 \cdot 92$ & $9,11,14,16$ \\
\hline $5 \cdot 35$ & $G R L$ & 1 & $0 \cdot 20$ & 0.29 & $5 q 11 \rightarrow q 13$ & 0.69 & $3 \cdot 46$ & 18 \\
\hline $6 \cdot 20$ & $H L A$ & 1 & $0 \cdot 10$ & $-6 \cdot 65$ & $6 p 21 \cdot 3$ & $14 \cdot 99$ & 29.98 & 8. 10 \\
\hline $6 \cdot 21$ & $B F$ & 1 & $(0 \cdot 10$ & $-1 \cdot 30$ & $6 \mathrm{p} 21 \cdot 3$ & $2 \cdot 93$ & $5 \cdot 86$ & 10 \\
\hline $6 \cdot 23$ & GLOI & 1 & $0 \cdot 10$ & $-3 \cdot 78$ & $6 \mathrm{p} 21 \cdot 31 \rightarrow \mathrm{p} 21 \cdot 1$ & $8 \cdot 52$ & $17 \cdot() 4$ & 9.10 .16 \\
\hline $6 \cdot 53$ & FI3A & 1 & $0 \cdot 10$ & -2.57 & $6 \mathrm{p} 23 \rightarrow \mathrm{qter}$ & $5 \cdot 79$ & $11 \cdot 58$ & 18 \\
\hline $6 \cdot 93$ & $P L G$ & 1 & $0 \cdot 10$ & $-5 \cdot 90$ & $6 \mathrm{q} 25 \rightarrow \mathrm{qter}$ & $13 \cdot 30$ & $26 \cdot 59$ & 18 \\
\hline $7 \cdot 30$ & $E G F R$ & 1 & $0 \cdot 10$ & -0.47 & $7 \mathrm{p} 13 \rightarrow \mathrm{pll}$ & 1.06 & $2 \cdot 12$ & 18 \\
\hline $7 \cdot 34$ & $\mathrm{NJ} 33 \cdot 2$ & 1 & $0 \cdot 10$ & $-0 \cdot 63$ & 7 pter $\rightarrow \mathrm{q} 22$ & 1.42 & $2 \cdot 84$ & 7 \\
\hline $7 \cdot 34$ & D7 & 1 & $(0 \cdot 10$ & $-4 \cdot 86$ & 7 pter $\rightarrow$ q22 & $10 \cdot 95$ & 21.91 & 20 \\
\hline $7 \cdot 62$ & COLIA2 & 1 & $0 \cdot 10$ & $-1 \cdot 85$ & $7 \mathrm{q} 21 \cdot 3 \rightarrow \mathrm{q} 22 \cdot 1$ & $4 \cdot 17$ & $8 \cdot 34$ & 22 \\
\hline $7 \cdot 72$ & $M e t D$ & 1 & $0 \cdot 10$ & $-0 \cdot 58$ & $7 \mathrm{q} 21 \rightarrow \mathrm{q} 3 \mathrm{i}$ & $1 \cdot 31$ & $2 \cdot 61$ & 7 \\
\hline $8 \cdot 29$ & PLAT & 1 & $(0 \cdot 10$ & $-1 \cdot 65$ & $8 \mathrm{pl} 12$ & $3 \cdot 72$ & $7 \cdot 44$ & 22. 23 \\
\hline 8.93 & $T G$ & 1 & $0 \cdot 10$ & $-6 \cdot 21$ & $8 \mathrm{q} 24$ & $14 \cdot 00$ & $27 \cdot 99$ & 7. 23 \\
\hline $8 \cdot 93$ & $M Y C$ & 1 & $(0 \cdot 10$ & -1.48 & $8 \mathrm{q} 24$ & $3 \cdot 34$ & 6.67 & 17 \\
\hline $9 \cdot 17$ & D9SI & 1 & $0 \cdot 10$ & $-1 \cdot 55$ & $9 \mathrm{pter} \rightarrow \mathrm{q} 11$ & $3 \cdot 49$ & 6.99 & 22 \\
\hline $9 \cdot 34$ & $O R M$ & 1 & $0 \cdot 10$ & -1.32 & $9 q$ & 2.98 & 5.95 & 18 \\
\hline 9.94 & $A B L$ & 1 & $0 \cdot 10$ & -0.59 & $9 q 34$ & $1 \cdot 33$ & $2 \cdot 66$ & 19 \\
\hline 9.94 & $A B O$ & $i$ & $0 \cdot 10$ & -7.97 & $9 q 34$ & $17 \cdot 96$ & 35.93 & 9.10 .16 \\
\hline 9.94 & $A K I$ & 1 & $0 \cdot 10$ & 0.49 & $9 q 34$ & 0.31 & $3 \cdot 07$ & 9 \\
\hline $11 \cdot 02$ & HRASI & 1 & $0 \cdot 10$ & $-10 \cdot 77$ & $11 \mathrm{p} 15 \cdot 5 \rightarrow$ pter & $24 \cdot 27$ & $48 \cdot 55$ & 7. 19,22 \\
\hline $11 \cdot 03$ & INS & 1 & $0 \cdot 10$ & $-5 \cdot 43$ & $11 \mathrm{p} 15 \cdot 5$ & $12 \cdot 24$ & $24 \cdot 48$ & 18. 22 \\
\hline $11 \cdot 07$ & $H b G I$ & 1 & $0 \cdot 10$ & -0.74 & 11 pl5 & 1.67 & $3 \cdot 34$ & 17 \\
\hline $11 \cdot 09$ & $C A L C l$ & 1 & $0 \cdot 10$ & -1.93 & $11 \mathrm{p} 15 \cdot 4 \longrightarrow \mathrm{p} 15 \cdot 1$ & $4 \cdot 35$ & $8 \cdot 70$ & 22 \\
\hline $11 \cdot 09$ & PTH & 1 & $0 \cdot 10$ & -0.67 & $11 \mathrm{p} 15 \cdot 4 \rightarrow \mathrm{p} 15 \cdot 1$ & $1 \cdot 51$ & $3 \cdot() 2$ & 17. 22 \\
\hline $11 \cdot 21$ & D1IS21 & 1 & $0 \cdot 10$ & $-1 \cdot 07$ & $11 \mathrm{p} 14 \longrightarrow \mathrm{p} 13$ & $2 \cdot 41$ & 4.82 & 21 \\
\hline $11 \cdot 23$ & D11S12 & 1 & $0 \cdot 10$ & $-1 \cdot 33$ & $11 \mathrm{p} 15 \cdot 5$ & $3 \cdot 00$ & $6 \cdot(00)$ & 22 \\
\hline $11 \cdot 27$ & $C A T$ & 1 & $0 \cdot 10$ & $-1 \cdot 80$ & $11 \mathrm{p} 13$ & $4 \cdot 06$ & $8 \cdot 11$ & 22 \\
\hline $11 \cdot 57$ & $D 11$ & 1 & $0 \cdot 10$ & $-2 \cdot 45$ & $11 \mathrm{p} 14 \rightarrow \mathrm{qter}$ & $5 \cdot 52$ & $11 \cdot(04$ & 20 \\
\hline $11 \cdot 74$ & $A P O A 4$ & 1 & $0 \cdot 10$ & $-1 \cdot 17$ & 11 q $13 \rightarrow$ qter & $2 \cdot 64$ & $5 \cdot 27$ & 18 \\
\hline $11 \cdot 85$ & ETS1 & 1 & $0 \cdot 10$ & $-4 \cdot 07$ & $11 q 23 \rightarrow q 24$ & $9 \cdot 17$ & $18 \cdot 35$ & 7. 17 \\
\hline $12 \cdot 19$ & KRAS2 & 1 & $0 \cdot 10$ & $-3 \cdot 18$ & $12 \mathrm{p} 12 \cdot 1$ & $7 \cdot 17$ & $14 \cdot 33$ & 19 \\
\hline $12 \cdot 31$ & $D 12 S 6$ & 1 & $(0 \cdot 10$ & $-2 \cdot(05$ & $12 \mathrm{cen} \rightarrow \mathrm{q} 13$ & $4 \cdot 62$ & 9.24 & 22 \\
\hline $12 \cdot 76$ & $I G F I$ & 1 & $0 \cdot 10$ & $-2 \cdot 01$ & $12 \mathrm{q} 22 \rightarrow \mathrm{q} 24 \cdot 1$ & $4 \cdot 53$ & $9 \cdot(06$ & 17. 19 \\
\hline $12 \cdot 81$ & $P A H$ & 1 & $0 \cdot 10$ & $-1 \cdot 64$ & $12 \mathrm{q} 22 \rightarrow \mathrm{q} 24 \cdot 2$ & $3 \cdot 70$ & $7 \cdot 39$ & 7 \\
\hline $13 \cdot 28$ & $E S D$ & 1 & $0 \cdot 10$ & $-3 \cdot 34$ & $13 q 14 \cdot 1$ & $7 \cdot 53$ & $15 \cdot 16$ & 9. 10 \\
\hline $13 \cdot 41$ & D1.3S5 & 1 & $0 \cdot 10$ & $-1 \cdot() 6$ & $13 \mathrm{q} 12 \rightarrow \mathrm{q} 22$ & $2 \cdot 39$ & $4 \cdot 78$ & 22 \\
\hline
\end{tabular}


TAPL.E 1 Continued.

\begin{tabular}{|c|c|c|c|c|c|c|c|c|c|}
\hline $\begin{array}{l}\text { Position } \\
\text { on the } \\
\text { chromosome }\end{array}$ & Locus & $\begin{array}{l}\text { Data } \\
\text { type }\end{array}$ & $\begin{array}{l}\text { Theta } \\
\text { (cM) }\end{array}$ & $\begin{array}{l}\text { Lod } \\
\text { score }\end{array}$ & Localisation & $r$ & $s$ & Reference & $\frac{\overline{\vec{C}}}{\overline{0}}$ \\
\hline $14 \cdot 88$ & $P I$ & 1 & $0 \cdot 10$ & $-7 \cdot 79$ & $14 \mathrm{q} 32 \cdot 1$ & $17 \cdot 56$ & $35 \cdot 11$ & 7. 16.18 & $\bar{n}$ \\
\hline 14.95 & IGHV & 1 & 0.10 & $-4 \cdot 11$ & $14 \mathrm{q} 32 \cdot 3$ & $9 \cdot 26$ & 18.53 & 7 & $\frac{\omega}{2}$ \\
\hline 14.95 & $G M$ & 1 & $0 \cdot 10$ & $-0 \cdot 27$ & $14 q 32 \cdot 3$ & 0.61 & $1 \cdot 22$ & 7 & \\
\hline $15 \cdot 32$ & DISSI & 1 & $0 \cdot 10$ & -1.81 & $15 q 14 \rightarrow q 21$ & $4 \cdot 08$ & $8 \cdot 16$ & 22 & \\
\hline $16 \cdot 14$ & $H B A$ & 1 & $0 \cdot 10$ & $-1 \cdot 35$ & $16 \mathrm{pl} 12$ & $3 \cdot 04$ & $6 \cdot 09$ & 7. 20.22 & \\
\hline $16 \cdot 19$ & $P G P$ & 1 & $0 \cdot 10$ & $-3 \cdot 15$ & $16 \mathrm{p} 11 \rightarrow$ pter & $7 \cdot 10$ & $14 \cdot 20$ & 9.10 .16 & \\
\hline $16 \cdot 77$ & $A P R T$ & $i$ & $0 \cdot 10$ & $-4 \cdot 59$ & $16 \mathrm{q} 22$ & $10 \cdot 34$ & $20 \cdot 69$ & 7. 21 & \\
\hline $16 \cdot 79$ & $H P$ & 1 & $0 \cdot 10$ & $-1 \cdot 65$ & $16 \mathrm{q} 22 \cdot 1$ & $3 \cdot 72$ & $7 \cdot 44$ & $9.10,16,21$ & \\
\hline $17 \cdot 06$ & $D I 7 S I$ & 1 & $0 \cdot 10$ & -0.22 & $17 \mathrm{pl} \rightarrow$ pter & 0.50 & 0.99 & 22 & \\
\hline $17 \cdot(16$ & $M Y H 2$ & $i$ & $0 \cdot 10$ & $-1 \cdot 10$ & $17 \mathrm{pl} 3 \rightarrow$ pter & $2 \cdot 48$ & 4.96 & 22 & \\
\hline $17 \cdot 32$ & $D 17 Z 1$ & 1 & $0 \cdot 10$ & $1 \cdot 17$ & $17 \mathrm{cen}$ & 0.73 & $7 \cdot 32$ & 22 & \\
\hline $17 \cdot 34$ & $E R B A 2$ & $i$ & $0 \cdot 10$ & $-0 \cdot 38$ & $17 \mathrm{ql1}$ & 0.86 & 1.71 & 20 & \\
\hline $19 \cdot 12$ & $\mathrm{C} 3$ & 1 & $0 \cdot 10$ & $-2 \cdot 59$ & $19 \mathrm{pl} 3 \cdot 3 \rightarrow \mathrm{pl} 3 \cdot 2$ & $5 \cdot 84$ & $11 \cdot 67$ & $9,13,16,21$ & i \\
\hline $19 \cdot 57$ & $A P O C 2$ & 1 & $0 \cdot 10$ & $-7 \cdot 55$ & $19 \mathrm{cen} \rightarrow \mathrm{q} 13.2$ & $17 \cdot(12$ & $34 \cdot(03$ & 9.21 & \\
\hline $19 \cdot 72$ & $S E$ & 1 & $0 \cdot 10$ & $-2 \cdot 32$ & $19 q$ & $5 \cdot 23$ & $10 \cdot 46$ & 9.13 & \\
\hline $20 \cdot 19$ & D20S5 & 1 & $0 \cdot 10$ & $-1 \cdot 20$ & $20 \mathrm{pl} 2$ & $2 \cdot 70$ & $5 \cdot 41$ & 7 & \\
\hline $22 \cdot 22$ & D20S6 & $i$ & $0 \cdot 10$ & -1.93 & $20 \mathrm{p}$ & $4 \cdot 35$ & $8 \cdot 70$ & 7 & $\rightarrow$ \\
\hline $20 \cdot 86$ & $D 20 S 4$ & $i$ & $0 \cdot 10$ & $-4 \cdot 47$ & $20 \mathrm{q} 13.2$ & $10 \cdot 07$ & $20 \cdot 15$ & 7. 22 & \\
\hline $21 \cdot 77$ & $D 21 S 17$ & 1 & $0 \cdot 10$ & $-0 \cdot 20$ & $21 \mathrm{q} 21 \cdot 2 \rightarrow \mathrm{qter}$ & 0.45 & 0.90 & 22 & \\
\hline $22 \cdot 37$ & $D 22 S 9$ & 1 & $0 \cdot 10$ & $-3 \cdot 59$ & $22 q 11$ & $8 \cdot(199$ & $16 \cdot 18$ & 19 & 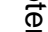 \\
\hline $22 \cdot 37$ & $I G L V$ & 1 & $0 \cdot 10$ & $-4 \cdot 13$ & $22 \mathrm{q} 11 \cdot 1 \rightarrow \mathrm{q} 11 \cdot 2$ & $9 \cdot 31$ & $18 \cdot 62$ & 7 & \\
\hline $22 \cdot 52$ & $D 22 S 1$ & 1 & $0 \cdot 10$ & -1.97 & $22 \mathrm{q} 11 \cdot 2 \rightarrow \mathrm{q} 13$ & $4 \cdot 44$ & $8 \cdot 88$ & 19. 22 & $\overline{8}$ \\
\hline 22.59 & $S I S$ & $i$ & $0 \cdot 10$ & -3.73 & $22 \mathrm{q} 12 \cdot 3 \rightarrow \mathrm{q} 13 \cdot 1$ & 8.41 & $16 \cdot 81$ & $15,19,20$ & \\
\hline
\end{tabular}

$\mathrm{r}=$ equivalent number of recombinants.

$\mathrm{s}=$ equivalent number of informative meioses.

TABLE 2 Linkage data between VRNF and those marker loci not accurately localised.

\begin{tabular}{|c|c|c|c|c|c|c|}
\hline $\begin{array}{l}\text { Chromosome } \\
\text { No }\end{array}$ & Locus & $\begin{array}{l}\text { Theta } \\
\text { (cM) }\end{array}$ & $\begin{array}{l}\text { Lod } \\
\text { score }\end{array}$ & $r$ & $s$ & Reference \\
\hline 1 & $D 1 S 2$ & $0 \cdot 10$ & $-1 \cdot(04$ & $2 \cdot 35$ & $4 \cdot 69$ & 22 \\
\hline 2 & $J K$ & $0 \cdot 10$ & -0.39 & 0.88 & 1.76 & 10.16 \\
\hline 3 & $A 2 H S$ & $0 \cdot 10$ & -2.59 & $5 \cdot 83$ & $11 \cdot 67$ & 18 \\
\hline 4 & $Y N H 24$ & $0 \cdot 10$ & $-2 \cdot 35$ & $5 \cdot 29$ & $10 \cdot 59$ & 22 \\
\hline $\begin{array}{l}6 \\
6\end{array}$ & $\begin{array}{l}D 6 \\
D 6 S 3\end{array}$ & $\begin{array}{l}0 \cdot 10 \\
0 \cdot 10\end{array}$ & $\begin{array}{l}-5 \cdot 32 \\
-0 \cdot 17\end{array}$ & $\begin{array}{r}11.99 \\
(0.39\end{array}$ & $\begin{array}{r}23.98 \\
0.77\end{array}$ & $\begin{array}{l}20 \\
22\end{array}$ \\
\hline 8 & PTHH5 & $0 \cdot 10$ & $-2 \cdot 74$ & $6 \cdot 17$ & $12 \cdot 35$ & 22 \\
\hline 12 & $D 12$ & $0 \cdot 10$ & $-4 \cdot 86$ & 10.96 & 21.91 & 20 \\
\hline $\begin{array}{l}17 \\
17 \\
17 \\
17 \\
17 \\
17\end{array}$ & $\begin{array}{l}\text { LDRI11 } \\
\text { LDR92 } \\
D 17 S 3 \\
D 17 S 2 \\
\text { PTHH59 } \\
\text { YNZ22 }\end{array}$ & $\begin{array}{l}0 \cdot 10 \\
0 \cdot 10 \\
0 \cdot 10 \\
0 \cdot 10 \\
0 \cdot 10 \\
0 \cdot 10\end{array}$ & $\begin{array}{l}-3 \cdot 01 \\
-0.71 \\
-0.56 \\
-2.40 \\
-3 \cdot 01 \\
-1.36\end{array}$ & $\begin{array}{l}6.79 \\
1.60) \\
1.26 \\
5 \cdot 41 \\
6 \cdot 79 \\
3 \cdot(16\end{array}$ & $\begin{array}{r}13 \cdot 57 \\
3 \cdot 20 \\
2 \cdot 52 \\
10 \cdot 82 \\
13 \cdot 57 \\
6 \cdot 13\end{array}$ & $\begin{array}{l}21 \\
21 \\
22 \\
22 \\
22 \\
22\end{array}$ \\
\hline 18 & D18S1 & $0 \cdot 10$ & $-2 \cdot(08$ & $4 \cdot 69$ & $9 \cdot 38$ & 22 \\
\hline 22 & $D 22 S 10$ & $0 \cdot 10$ & $-0 \cdot 23$ & 0.52 & $1 \cdot 04$ & 20 \\
\hline $\begin{array}{l}? \\
? \\
? \\
? \\
? \\
?\end{array}$ & $\begin{array}{l}\text { GPT } \\
P T H 162 \\
P T H H 39 \\
Y N Z 132 \\
P \\
\text { C6 }\end{array}$ & $\begin{array}{l}0 \cdot 10 \\
0 \cdot 10 \\
0 \cdot 10 \\
0 \cdot 10 \\
0 \cdot 10 \\
0 \cdot 10\end{array}$ & $\begin{array}{l}-0.30 \\
-2.13 \\
-0.76 \\
-0.87 \\
-0.07 \\
-2.38\end{array}$ & $\begin{array}{l}0.68 \\
4 \cdot 80 \\
1.72 \\
1.96 \\
0 \cdot 16 \\
5.37\end{array}$ & $\begin{array}{r}1.35 \\
9.60 \\
3.43 \\
3.92 \\
0 \cdot 32 \\
10 \cdot 73\end{array}$ & $\begin{array}{l}16 \\
22 \\
22 \\
22 \\
16 \\
7.18\end{array}$ \\
\hline
\end{tabular}

$\mathbf{r}=$ equivalent number of recombinants.

$\mathrm{s}=$ equivalent number of informative meioses. 
TABLE 3 Results from programme EXCLUDE, indicating the most likely location of the VRNF gene.

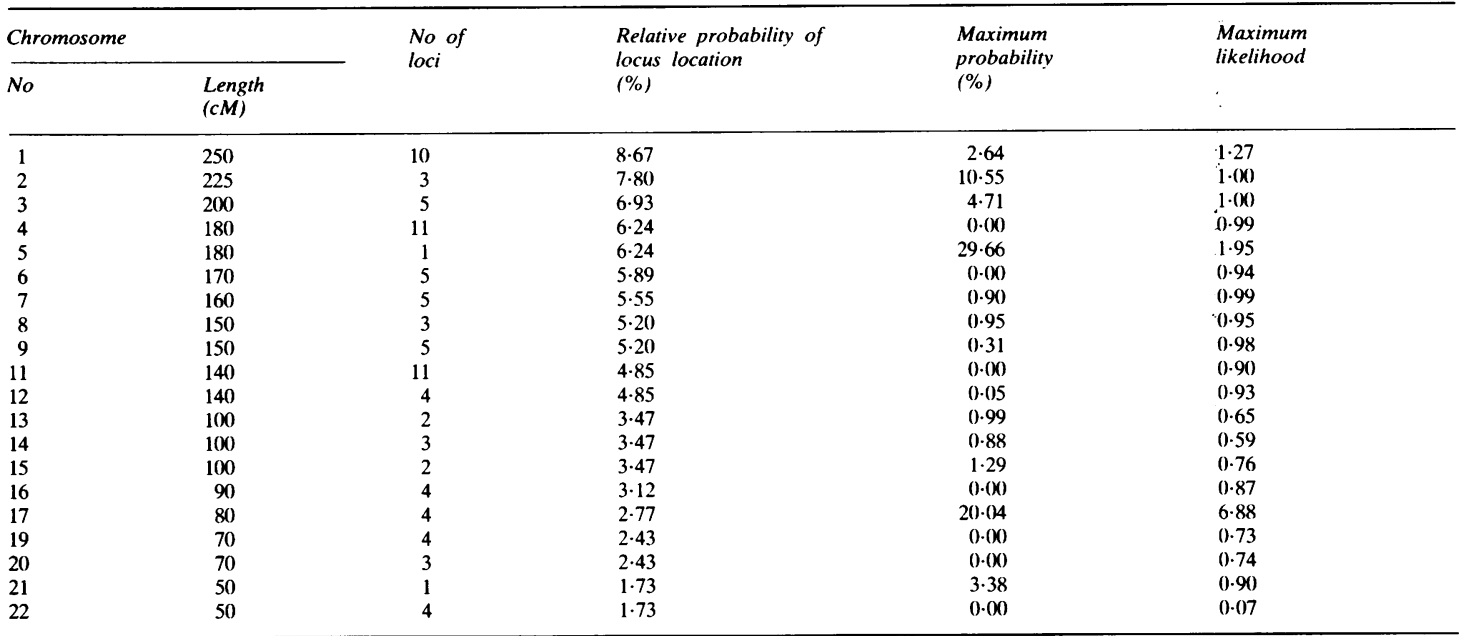

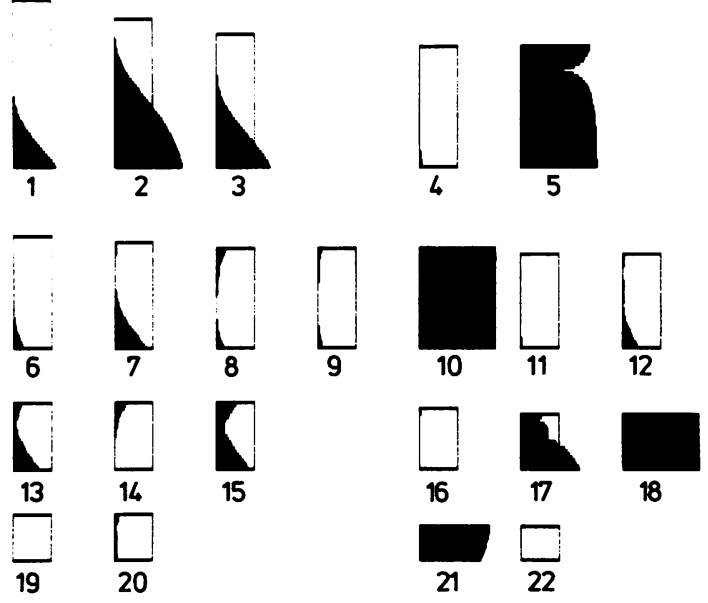

FIGURE The exclusion map for VRNF. The shaded area of the chromosome shows the possible location of the disease gene and the white area the excluded zones.

\section{Results and discussion}

The additional data presented in this issue of the Journal provide the means of extending the exclusion map for the VRNF locus. Before this consortium, data on a total of 28 linkage markers on 13 chromosomes were available, including some of our own unpublished data set. ${ }^{7}$ This has now been expanded to 114 and at least one marker has been studied on all chromosomes except No 10 (table 4).
Although one RFLP has been studied on chromosome 18 , it has no subchromosomal localisation and therefore could not be included in the exclusion map; taken together, these two chromosomes represent about $8 \%$ of the genome. The overall data show that the most likely location for the VRNF gene is on chromosome 17 with a likelihood of seven times more likely than being on any other chromosome. Another possible chromosome is No 5, with a likelihood of only 2 . As this exclusion map clearly shows, the chance of the disease gene being on any other chromosome is very remote.

The strong indication from the exclusion map that the VRNF gene lies on one of the chromosomes 5 , 10,17 , or 18 will give impetus to the use of markers on those chromosomes, and to the search for new informative polymorphisms in these areas of the genome. The possibility must also be considered, however, that genetic heterogeneity might mask a positive result. While the existence of a number of large individual kindreds in the various studies makes this unlikely, a careful analysis for heterogeneity would be required in the event of negative results for those chromosomes not currently excluded.

We would like to thank the British Neurofibromatosis Patients' Association, LINK, for organising the European Symposium on Neurofibromatosis, members of the consortium who provided us with their data for this analysis, and Professor Marcus Pembrey who chaired and coordinated the consortium. 
TABLE 4 Comparison of available linkage data on VRNF before and after the collaborative work of this consortium. $\stackrel{\overparen{D}}{\Delta}$

\begin{tabular}{|c|c|c|c|c|c|c|c|c|}
\hline \multicolumn{2}{|c|}{ Chromosome } & \multicolumn{3}{|c|}{ Before consortium } & \multicolumn{3}{|c|}{ After consortium } & $\bar{\omega}$ \\
\hline \multirow[t]{2}{*}{ No } & \multirow{2}{*}{$\begin{array}{l}\text { Length } \\
(c M)\end{array}$} & \multirow{2}{*}{$\begin{array}{l}\text { No of } \\
\text { loci }\end{array}$} & \multicolumn{2}{|c|}{ Exclusion area } & \multirow{2}{*}{$\begin{array}{l}\text { No of } \\
\text { loci }\end{array}$} & \multicolumn{2}{|c|}{ Exclusion area } & \multirow{2}{*}{$\begin{array}{l}\text { ত } \\
\text { ত } \\
\overline{\bar{\sigma}}\end{array}$} \\
\hline & & & $(c M)$ & $(\%)$ & & $(c M)$ & $(\%)$ & \\
\hline 1 & 250 & 5 & $245 \cdot 2$ & $8 \cdot 50$ & 10 & $243 \cdot 4$ & $8 \cdot 44$ & \\
\hline 2 & 225 & 1 & $204 \cdot 7$ & $7 \cdot 10$ & 3 & $201 \cdot 3$ & 6.98 & \\
\hline 3 & 200 & 1 & $183 \cdot 0$ & $6 \cdot 34$ & 5 & 19()$\cdot 6$ & $6 \cdot 61$ & $\stackrel{\mathscr{0}}{\mathscr{C}}$ \\
\hline 4 & 180 & 2 & $179 \cdot 0$ & $6 \cdot 20$ & 11 & $18(0 \cdot 0$ & $6 \cdot 24$ & \\
\hline 5 & 180 & - & $0 \cdot 0$ & $0 \cdot(00$ & 1 & $126 \cdot 6$ & $4 \cdot 39$ & 0 \\
\hline 6 & 170 & 3 & $164 \cdot 8$ & $5 \cdot 71$ & 5 & $170 \cdot 0$ & $5 \cdot 89$ & \\
\hline 7 & 160 & 1 & $150 \cdot 0$ & $5 \cdot 20$ & 5 & $158 \cdot 6$ & $5 \cdot 50$ & $\overrightarrow{\overrightarrow{1}}$ \\
\hline 8 & 150 & - & $0 \cdot 0$ & $0 \cdot(0)$ & 3 & $148 \cdot 6$ & $5 \cdot 15$ & $\omega$ \\
\hline 9 & 150 & 1 & $144 \cdot 6$ & $5 \cdot() 1$ & 5 & $149 \cdot 5$ & $5 \cdot 18$ & ? \\
\hline 10 & 150 & - & $0 \cdot 0$ & $0 \cdot(0)$ & - & $0 \cdot 0$ & 0.00 & 3 \\
\hline 11 & 140 & - & $0 \cdot 0$ & $0 \cdot(0)$ & 11 & $140 \cdot 0$ & $4 \cdot 85$ & बै \\
\hline 12 & 140 & - & $0 \cdot 0$ & 0.00 & 4 & $139 \cdot 9$ & $4 \cdot 85$ & N \\
\hline 13 & 100 & 1 & $99 \cdot 2$ & 3.44 & 2 & $99 \cdot 0$ & 3.43 & $A$ \\
\hline 14 & 100 & 2 & $98 \cdot 3$ & 3.41 & 3 & $99 \cdot 1$ & 3.44 & ق \\
\hline 15 & 100 & - & $0 \cdot 0$ & 0.00 & 2 & 98.7 & 3.42 & i \\
\hline 16 & 90 & 3 & 89.7 & $3 \cdot 11$ & 4 & $90 \cdot()$ & $3 \cdot 12$ & $\overrightarrow{\mathrm{c}}$ \\
\hline 17 & 80 & - & $0 \cdot 0$ & $0 \cdot(0)$ & 4 & $64 \cdot()$ & $2 \cdot 22$ & \\
\hline 18 & 80 & - & $0 \cdot 0$ & $0 \cdot(0)$ & - & $0 \cdot 0$ & 0.00 & \\
\hline 19 & 70 & 3 & $70 \cdot()$ & $2 \cdot 43$ & 4 & $70 \cdot(0$ & $2 \cdot 43$ & $\rightarrow$ \\
\hline 20 & 70 & 3 & $70 \cdot()$ & $2 \cdot 43$ & 3 & $70 \cdot 0$ & $2 \cdot 43$ & $c 0$ \\
\hline 21 & 50 & - & $0 \cdot 0$ & $0 \cdot(00)$ & 1 & $48 \cdot 3$ & 1.67 & \\
\hline 22 & 50 & 2 & $50 \cdot 0$ & 1.73 & 4 & $50 \cdot 0$ & 1.73 & Оृ \\
\hline Total & 2885 & 28 & $1748 \cdot 4$ & $60 \cdot 60)$ & 90 & $2537 \cdot 6$ & 87.96 & 3 \\
\hline
\end{tabular}

\section{References}

' Riccardi VM, Carey JC. Von Recklinghausen neurofibromatosis and genetic linkage studies: clinical considerations. $J$ Med Genet 1987;24:521-2.

${ }^{2}$ Huson S, Upadhyaya M, Sarfarazi M, Edwards J, Harper PS. Peripheral neurofibromatosis: progress in mapping of the disease locus. J Med Genet 1987;24:246A.

${ }^{3}$ Edwards JH. Exclusion mapping. J Med Genet 1987;24: 539-43.

${ }^{4}$ Cook PJL, Noades JE, Lomas CG, Buckton KE, Robson EB. Exclusion mapping illustrated by the MNSs blood group. Ann Hum Genet 1980;44:61-73.

5 Edwards JH. The analysis of X linkage. Ann Hum Genet 1971:34:229-50.

${ }^{6}$ Morton NE. Sequential tests for the detection of linkage. Am J Hum Genet 1955;7:277-318.

7 Upadhyaya M, Sarfarazi M, Huson SM, Harper PS. Further exclusion data for the Von Recklinghausen neurofibromatosis gene: a genetic linkage study of 19 polymorphic markers. J Med Genet 1987;24:534-6.

${ }^{8}$ Lepage V, Tongio MM, Mayer S, et al. Neurofibromatosis (Von Recklinghausen disease). In: Terasaki PI, ed. Histocompatibility testing 1980. Los Angeles: UCLA Tissue Laboratory, 1981:719-21.

y Dunn BG, Ferrell R, Riccardi V. A genetic linkage study of 15 families with Von Recklinghausen neurofibromatosis. Am J Med Genet 1985;22:403-7.

1" Spence MA, Bader JL, Parry DM, et al. Linkage analysis of neurofibromatosis (Von Recklinghausen discase). J Med Genet 1983;20:334-7.

"Spence MA, Parry DM, Bader JL, et al. Genetic linkage analysis of neurofibromatosis. Ann NY Acad Sci 1986:486:287-92.

12 Darby JK, Goslin K, Riccardi VM, et al. Linkage analysis between B-nerve growth factor gene and other chromosome 1p markers and disseminated neurofibromatosis. Ann NY Acad Sci 1986;486:311-26.

${ }^{13}$ Huson SM. Meredith AL, Sarfarazi M, Shaw DJ, Compston DAS, Harper PS. Linkage analysis of peripheral neurofibroma- tosis (Von Recklinghausen disease) and chromosome 19 ngar kers linked to myotonic dystrophy. J Med Genet 1986;23:557. V

14 Dietz JN, Robbins T, Cannon LA, et al. Linkage analysis of Recklinghausen neurofibromatosis: chromosome 4 and $\bar{q} 90$ Genet Epidemiol 1986;3:313-21.

15 Seizinger BR, Tanzi RE, Gilliam TC, et al. Genetic linkag analysis of neurofibromatosis with DNA markers. Ann $N$ kू Acad Sci 1986;486:304-10.

${ }^{16}$ Spence MA, Sparkes RS, Parry DM, Bale SR, Cortessis V尺 Mulvihill JJ. Genetic linkage studies with neurofibromatosis the question of heterogeneity. J Med Genet 1987;24:527-9. 윽

${ }^{17}$ Kittur S, Lubs ML, Bauer M, Chakravarti A, Kazazian $\mathrm{H}_{3}^{3}$ Linkage analysis of neurofibromatosis. $J$ Med Gene 1987;24:526-7.

18 Ferrell RE, Buetow KH, Darby JK, et al. Von Recklinghauseñ neurofibromatosis: a linkage study of candidate and randon marker genes. J Med Genet 1987;24:522-4.

14 Seizinger BR, Rouleau G, Lane AH, et al. DNA linkagé analysis in Von Recklinghausen neurofibromatosis. J Med Genef 1987:24:529-30.

20) Mathew CGP, Thorpe K, Easton DF, et al. Linkage analysis o\$ British and Indian families with Von Recklinghausen neurofibromatosis. J Med Genet 1987:24:524-6.

${ }^{21}$ Pericak-Vance MA. Yamaoka LH, Vance JM, et al. Linkaga studies in peripheral neurofibromatosis. $J$ Med Gene 1987:24:530-2.

22 Barker D, Wright E, Nguyen K, et al. A genomic search fof linkage of neurofibromatosis to RFLPs. J Med Genet 1987:24:536-8.

23 Diehl SR, Boehnke M, Collins FS, et al. Linkage analysis oर́ peripheral neurofibromatosis to DNA markers on chromosome 8. J Med Genet 1987;24:532-4.

Correspondence and requests for reprints to $\operatorname{Dr} \mathrm{M}$ Sarfarazi, Institute of Medical Genetics, University of Wales College of Medicine, Heath Park, Cardiff CF4 4XN. 